\title{
Identification of the lipid biomarkers from plasma in idiopathic pulmonary fibrosis by Lipidomics
}

\author{
Feng Yan $^{1 *+}$, Zhensong Wen ${ }^{2+}$, Rui Wang ${ }^{3}$, Wenling Luo ${ }^{1}$, Yufeng $\mathrm{Du}^{4}$, Wenjun Wang ${ }^{5}$ and Xianyang Chen ${ }^{5}$
}

\begin{abstract}
Background: Idiopathic pulmonary fibrosis (IPF) is an irreversible interstitial pulmonary disease featured by high mortality, chronic and progressive course, and poor prognosis with unclear etiology. Currently, more studies have been focusing on identifying biomarkers to predict the progression of IPF, such as genes, proteins, and lipids. Lipids comprise diverse classes of molecules and play a critical role in cellular energy storage, structure, and signaling. The role of lipids in respiratory diseases, including cystic fibrosis, asthma and chronic obstructive pulmonary disease (COPD) has been investigated intensely in the recent years. The human serum lipid profiles in IPF patients however, have not been thoroughly understood and it will be very helpful if there are available molecular biomarkers, which can be used to monitor the disease progression or provide prognostic information for IPF disease.
\end{abstract}

Methods: In this study, we performed the ultraperformance liquid chromatography coupled with quadrupole time of flight mass spectrometry (UPLC-QTOF/MS) to detect the lipid variation and identify biomarker in plasma of IPF patients. The plasma were from 22 IPF patients before received treatment and 18 controls.

Results: A total of 507 individual blood lipid species were determined with lipidomics from the 40 plasma samples including 20 types of fatty acid, 159 types of glycerolipids, 221 types of glycerophospholipids, 47 types of sphingolipids, 46 types of sterol lipids, 7 types of prenol lipids, 3 types of saccharolipids, and 4 types of polyketides. By comparing the variations in the lipid metabolite levels in IPF patients, a total of 62 unique lipids were identified by statistical analysis including 24 kinds of glycerophoslipids, 30 kinds of glycerolipids, 3 kinds of sterol lipids, 4 kinds of sphingolipids and 1 kind of fatty acids. Finally, 6 out of 62 discriminating lipids were selected as the potential biomarkers, which are able to differentiate between IPF disease and controls with ROC analysis.

Conclusions: Our results provided vital information regarding lipid metabolism in IPF patients and more importantly, a few potentially promising biomarkers were firstly identified which may have a predictive role in monitoring and diagnosing IPF disease.

Keywords: Idiopathic pulmonary fibrosis, Plasma, Lipid, Lipidomics, Biomarkers

\section{Background}

Idiopathic pulmonary fibrosis (IPF) is a disease featured as chronic, progressive, irreversible interstitial pneumonia with a poor prognosis of unknown etiology [1] and a median survival of 3 to 5 years after initial diagnosis $[2,3]$. It more commonly occurs in patients of 50 to 70 years of

\footnotetext{
* Correspondence: yanfengyixue@163.com

Zhensong Wen is also a first co-author

${ }^{\dagger}$ Equal contributors

'Department of Respiration, First Hospital of Tsinghua University, Beijing 100016, China

Full list of author information is available at the end of the article
}

age. This disease is characterized by the histological pattern of usual interstitial pneumonia $[4,5]$ with parenchymal fibrosis and excess collagen deposition [6]. It is well accepted that the pathology of this disease includes fibroblast/myofibroblast proliferation, activation of alveolar epithelial cells, with exacerbated deposit of extracellular matrix leading to the gradual destruction of the lung tissue [7]. Although there have been increasing number of studies investigating the pathogenesis of idiopathic pulmonary fibrosis (IPF), the lacking of effective treatments and early diagnostic tools indicate the urgent needs for reliable biomarkers in both 
diagnosing at early stage and monitoring the progression of this disease. In the recent publications, a few potentially useful blood cellular and molecular biomarkers have been identified, including chemokines, proteases and growth factors [8]. Despite the recent progress, there has not been a single biomarker proved to be useful in diagnosing and monitoring the progress of IPF. The diagnosis of IPF has not changed much over the last few years, which means that a multi-disciplinary approach is probably required for a breakthrough. With the new emergent technology of lipidomics, to identify novel lipid biomarkers for diagnostic and monitor purposes of IPF becomes possible.

Lipids comprise diverse classes of molecules that play a critical role in cellular energy storage, structure, and signaling [9-11]. Previously, lipids are only considered as the components of membranes and source. Now lipids are known to act as a indispensable factor in the immune response by organizing signaling complexes in cellular membrane, such as lipid rafts [12] or affecting the immune reaction by release of lipid-derived mediators $[13,14]$. The etiologies of certain diseases have been proven to be associated with individual lipid molecules and many studies have indicated that certain lipid metabolic disorders or abnormalities can lead to a variety of human diseases [10, 15-19].

The role of lipids in lung and respiratory diseases has attracted more attention in recent years including cystic fibrosis, asthma and COPD which are all associated with abnormal metabolism. For example, the epithelium lipid metabolism has been proved to be changed in asthmatic patients $[20,21]$. The amount of ceramides in the airway epithelium of a guinea pig model was found to be increased in response to the induction of experimental allergic asthma [22]. In another study, the increased amount of ceramides was detected in the airway epithelium and has been linked to cell death, infection susceptibility and immune inflammation in cystic fibrosis [23]. When it comes to IPF however, the human plasma lipid profiles of IPF is so far poorly understood and to identify reliable and unique lipid molecular biomarkers will be very beneficial in IPF diagnosis and management [24]. Based on the above reasons, to analyze and identify potential IPF-specific lipid biomarkers will contribute significantly to the diagnosis and management in IPF patients.

With the development of omicis [25] and the advanced mass spectrometry have made it feasible to identify and quantify a variety of lipids species in human samples such as the tandem mass spectrometer utilized (MS/MS) [26, 27], direct infusion MS (DIMS) [28], and liquid chromatography-mass spectrometry (LC-MS) [29-32]. Among different LC-MS platforms, ultraperformance liquid chromatography coupled with quadrupole time of flight mass spectrometry (UPLC-QTOF/MS) is widely adapted to lipidomics due to its enhanced reproducibility of retention time [33-35]. In this study, a global lipid profiling was performed containing measurement of 20 kinds of fatty acid, 159 kinds of glycerolipids, 221 kinds of glycerophospholipids, 47 kinds of sphingolipids, 46 kinds of sterol lipids, 7 kinds of prenol lipids, 3 kinds of saccharolipids, and 4 kinds of polyketides. Subsequently, the correlation analysis, receiver operating characteristic (ROC) analysis and orthogonal partial least squares discriminant analysis (OPLS-DA) were performed to evaluate the variations in lipid metabolites. The potential influence of gender, smoking history, and disease stages on the lipid metabolites was also looked at between IPF object and/or controls. Our results provided vital information regarding lipid metabolism in IPF patients and more importantly, a few potentially promising biomarkers were firstly identified which may have a predictive role in monitoring and diagnosing IPF disease.

\section{Methods \\ Sample and collection}

In this study, 22 IPF patients and 18 controls were obtained from the First Hospital of Tsinghua University from January 2014 to March 2016. All IPF patients were diagnosed with IPF after the age of 60 , and all of controls were all above 60 years old when examined. The demographic figures are listed in the Table 1 . The stages of disease are classified into mild and severe according to the arterial blood oxygen partial pressure $(\geqq$ $60 \mathrm{mmHg}$ ). The diagnosis of IPF patients was made according to the internal recommendations of the ATS/ERS/JRS/ALAT statement using high-resolution computed tomography (HRCT), as well as the clinical history of the patient [36]. All cases were discussed in our discussion team about interstitial lung disease composed of: a specialist in pulmonary rehabilitation, a specialist in occupational medicine, a radiologist, a rheumatologist, a pulmonologist and a pathologist. Blood samples were collected from each patient before received treatment and control. The controls were precluded if there was a history of pulmonary disease. For each subject, $10 \mathrm{ml}$ whole blood was collected into a vessel tube containing heparin as anticoagulant. Each sample was centrifuged at $1500 \mathrm{x} \mathrm{g}$ for $15 \mathrm{~min}$ to collect serum and stored at $-80{ }^{\circ} \mathrm{C} \mathrm{im}$ mediately until further analysis.

Table 1 Sample demographics of the study subjects

\begin{tabular}{lll}
\hline & IPF $(N=22)$ & Control $(N=18)$ \\
\hline Age, mean & 73 & 72 \\
Gender (males/females) & $11 / 11$ & $8 / 10$ \\
Smoking History (Yes/No) & $9 / 13$ & $10 / 8$ \\
Stages (mild/severe) & $11 / 11$ & - \\
\hline
\end{tabular}




\section{Lipidomics}

Liquid chromatography-mass spectrometry (LC-MS)-grade isopropanol, acetonitrile, methanol and water were purchased from Fisher Scientific (New Jersey, USA). Debrisoquine, Pro-Asn, glycoursodeoxycholic acid andmalic acid, 4-nitrobenzoic acid (4-NBA) were products of Sigma (St. Louis, MO, USA). High purity formic acid (99\%) was provided by Thermo-Scientific (Rockford, IL). The serum lipid extraction was conducted as described previously [37]. Briefly, the plasma samples were thawed on ice before being vortexed. For metabolite extraction, $25 \mu \mathrm{L}$ of plasma sample was mixed with $175 \mu \mathrm{L}$ of extraction buffer $(25 \%$ acetonitrile in $40 \%$ methanol and $35 \%$ water). The sample was then incubated on ice for $10 \mathrm{~min}$ before centrifuged again at $14,000 \mathrm{rpm}$ at $4{ }^{\circ} \mathrm{C}$ for $20 \mathrm{~min}$. Subsequently, the produced supernatant was transferred to a fresh tube and dried under vacuum. After drying, the dried samples were reconstituted in $200 \mu \mathrm{L}$ of buffer containing 5\% methanol, $1 \%$ acetonitrile and $94 \%$ water. Fine particles were removed by centrifuge at $13,000 \mathrm{rpm}$ for $20 \mathrm{~min}$ at $4{ }^{\circ} \mathrm{C}$. Finally, the supernatant was transferred to a glass vial for ultraperformance liquid chromatography-quadrupole time-of-flight mass spectrometry (UPLC-QTOF-MS) analysis.

\section{Statistical analysis}

All statistical analyses were conducted using $\mathrm{R}$ software version 2.9.1. The OPLS score plots and T-test and variable importance for projection (VIP) statistics were used to select significant variables leading to group separation. A supervised OPLS analysis was applied in our study to identify potential lipids that were used to classify the samples and remove non-correlated variables. The differences between the intensities of lipids in IPF and healthy controls were compared by $\mathrm{T}$ test when the data follow a normal distribution or Wilcoxon rank-sum test when otherwise. In this study, the identified lipids were pre-selected as potential biomarkers when VIP value is bigger than 1.0. To analyze the diagnostic value of potential lipid biomarkers for identifying IPF disease, a ROC analysis was performed. Correlation analysis of differential lipids was performed by MetaboAnalyst software. The influence of gender, smoking history, and stages of disease of individual subjects on lipid metabolism were evaluated by the Mann-Whitney $U$-test. The $p$ values of less than 0.05 was considered statistically significant.

\section{Ethics statement}

The clinical IPF samples included in this study were collected from the First hospital of Tsinghua University. All patient data were anonymous, so informed consent for participation was not required. The use of these samples was approved by the Institutional Review Board for human studies at the First Hospital of Tsinghua University.
In this study, all personal information including name, date of birth, and contact information was all de-identified and not disclosed.

\section{Results \\ IPF plasma lipid profiles of $\mathbf{5 0 7}$ apparent lipid species}

In this study, the plasma lipid profiles of 507 individual lipid species were determined with lipidomics from 40 plasma samples; 22 from IPF patients with 18 controls (Additional file 1: Table S1). The detected individual plasma apparent lipid species were classified into 8 categories: fatty acid, glycerolipid, glycerophospholipid, sphingolipid, sterol lipid, prenol lipid, saccharolipid, and polyketide in accordance with NIH-funded Consortium, which has built an ongoing website tools offering precise information based on numerous lipidomics studies [38]. The 507 individual lipid species included 20 kinds of fatty acyls (3.94\%), 159 kinds of glycerolipids (31.36\%), 221 kinds of glycerophospholipids (43.59\%), 47 kinds of sphingolipids (9.27\%), 46 kinds of sterol lipids (9.07\%), 7 kinds of prenol lipids (1.38\%), 3 kinds of saccharolipids $(0.59 \%)$, and 4 kinds of polyketides $(0.79 \%)$, respectively. The significant differences of each individual apparent lipid species between IPF patients and control groups were detailed in the context of this study.

\section{Statistical analysis of the lipid profiling}

Supervised orthogonal partial least squares (OPLS) analysis identifed the biggest variation in lipid profiling using a few orthogonal latent variables and was performed with the lipid-obtained data on the plasma in positive ion mode by the UPLC-QTOF-MS/MS. The metabolic patterns were plotted by the OPLS-DA model (Fig. 1A). The OPLS-DA model was used to unfold the difference of plasma metabolic pattern between IPF patients and control group. The OPSL score plot revealed the significant deviation between IPF patients and controls.

In order to identify the potential biomarkers, the S-plot analysis was used based on the plasma lipid profiling data (Fig. 1B). By comparing changes in the lipid metabolite levels of IPF patients, a total of 62 unique lipids were detected [variable importance for projection (VIP $>1$ ), Table 2]. The lipids were identified according to the reported methods as described previously [37]. They included 24 kinds of glycerophoslipids, 30 kinds of glycerolipids, 3 kinds of sterol lipids, 4 kinds of sphingolipids and 1 kind of fatty acid. The above lipids were selected as potential biomarkers from the S-plot for further analysis in our study.

All the determined glycerophospholipids and sphingolipids showed a decreasing tendency in IPF objects (Table 2). Although all of glycerophospholipids and sphingolipids also showed a similar decreasing pattern 


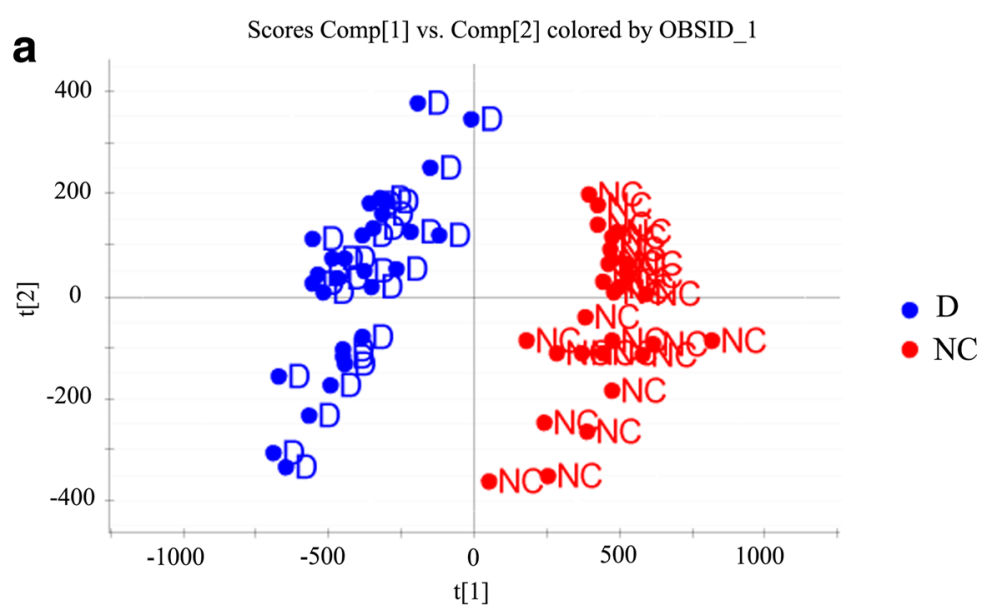

EZInfo controlled by Progenesis QI 3.0.1 -cc111.usp (M1:PCA-X) - 2016-12-16 09:13:00 (UTC+8)

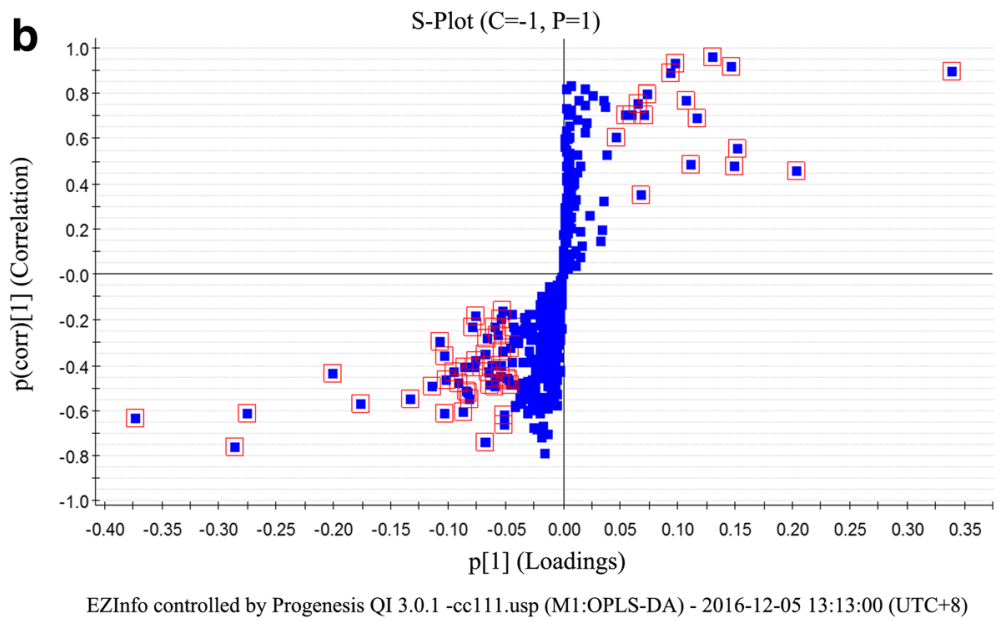

Fig. 1 a. OPLS-DA scores plot based on the plasma lipid profiling of IPF patients $(\bullet D)$ and controls $(\bullet N C)$. b. S-plot used in the lipid biomarkers selection. The lipids marked ( $\square$ ) are the lipids selected as potential biomarkers

in IPF patients, the magnitude of their drop was not the same. 15 out of 30 glycerolipids and 1 out of sterol lipids were lower in IPF objects. On the other hand, the remaining glycerolipids, sterol lipids and fatty acids had an increased level in IPF objects. These findings suggested that the observed changes in lipid profiles were likely caused by different expression in IPF patients.

\section{Correlation and receiver operating characteristic (ROC) curve analysis}

To better understand the relationship of metabolite differences with IPF disease, correlation analysis was applied to analyze these identified lipids data (Fig. 2). Firstly, The differences between the intensities of 62 identified lipids in IPF and healthy controls were compared by $\mathrm{T}$ test when the data follow a normal distribution or Wilcoxon rank-sum test when otherwise. Comprehensive analysis with $P$-value, 35 out of 62 identified lipids were selected to further analyze (Table 2, R1 to R35). Then, we evaluated the correlation between IPF disease and 35-selected lipids. As shown in Fig.2, the close correlations can be detected between IPF disease and 12 out of 35 identified lipids, including TG(16:0/18:2/18:2)[iso3] (R2), $\mathrm{PC}(22: 6 / 16: 0)$ (R3), Stigmasteryl ester(16:1) (R7) and (E,E)-3,7,11-Trimethyl2,6,10-dodecatrienyl dodecanoate (R9), DG(O-16:0/18:1) (R13), 3-Deoxyvitamin D3 (R16), TG(18:4/20:3/22:0)[iso6] (R17), DG(18:0/18:2/0:0)[iso2] (R21), DG(16:0/18:2/ 0:0)[iso2] (R22), DG(18:1/18:3/0:0)[iso2] (R24), TG(19:1/ 22:6/22:6)[iso3] (R25), and DG(18:1/18:2/0:0)[iso2] (R26) (correlation absolute value $>0.7$ ). This result indicates that these individual lipid molecules potentially are useful to differentiated the IPF patients from control group and the accuracy and efficiency possibly can be further increased if more lipid candidates are to be used.

Theoretically, those lipid molecules possessing close correlation with IPF disease would be more promising to be used as biomarkers. To further demonstrate the ability of the 12 out of 35 identified lipids to identify the 
Table 2 List of assigned statistically significantly lipid molecules and change trend of biomarkers on plasma lipid profile in positive ion mode after comparison of IPF patient samples to control samples

\begin{tabular}{|c|c|c|c|c|c|c|c|c|c|c|}
\hline No. & Code & $\begin{array}{l}\text { Calc m/ } \\
z\end{array}$ & $t_{R}$ & VIP & Trend $^{a}$ & Accepted Description & Lipidlon & Element & Lipid class & $P$-Value \\
\hline 1 & R1 & 1516.124 & 5.06 & 8.58719 & $\downarrow$ & $\begin{array}{l}C L\left(1^{\prime}-[20: 0 / 20: 0], 3^{\prime}-\right. \\
[18: 1 / 18: 2])\end{array}$ & $\mathrm{M}+\mathrm{H}, \mathrm{M}+\mathrm{Na}, \mathrm{M}+\mathrm{H}+\mathrm{Na}$ & $\mathrm{C}_{85} \mathrm{H}_{160} \mathrm{O}_{17} \mathrm{P}_{2}$ & Glycerophospholipids & $\begin{array}{l}7.90 \mathrm{E}- \\
08^{* * *}\end{array}$ \\
\hline 2 & R2 & 872.769 & 14.54 & 7.63644 & $\uparrow$ & $\begin{array}{l}\text { TG(16:0/18:2/ } \\
\text { 18:2)[iso3] }\end{array}$ & $\mathrm{M}+\mathrm{H}, \mathrm{M}+\mathrm{NH}_{4}$ & $\mathrm{C}_{55} \mathrm{H}_{98} \mathrm{O}_{6}$ & Glycerolipids & $\begin{array}{l}1.24 \mathrm{E}- \\
07^{* * *}\end{array}$ \\
\hline 3 & R3 & 806.567 & 4.52 & 6.33481 & $\downarrow$ & $P C(22: 6 / 16: 0)$ & $\begin{array}{l}\mathrm{M}+\mathrm{H}, \mathrm{M}+\mathrm{Na}, \mathrm{M}+\mathrm{K}, 2 \mathrm{M}+\mathrm{H} \\
2 \mathrm{M}+\mathrm{Na}\end{array}$ & $\mathrm{C}_{46} \mathrm{H}_{80} \mathrm{NO}_{8} \mathrm{P}$ & Glycerophospholipids & $\begin{array}{l}9.24 \mathrm{E}- \\
07^{* * *}\end{array}$ \\
\hline 4 & - & 877.724 & 14.55 & 6.05829 & $\downarrow$ & $\begin{array}{l}\text { TG(16:1/18:1/ } \\
\text { 18:2)[iso6] }\end{array}$ & $\mathrm{M}+\mathrm{Na}, \mathrm{M}+\mathrm{K}, 2 \mathrm{M}+\mathrm{Na}$ & $\mathrm{C}_{55} \mathrm{H}_{98} \mathrm{O}_{6}$ & Glycerolipids & 0.2370 \\
\hline 5 & - & 874.785 & 14.82 & 4.60354 & $\uparrow$ & $\begin{array}{l}\text { TG(16:0/18:1/ } \\
\text { 18:2)[iso6] }\end{array}$ & $\begin{array}{l}\mathrm{M}+\mathrm{H}-\mathrm{H}_{2} \mathrm{O}, \mathrm{M}+\mathrm{H}, \mathrm{M}+\mathrm{NH}_{4} \\
\mathrm{M}+\mathrm{Na} \\
\mathrm{M}+\mathrm{K}, 2 \mathrm{M}+\mathrm{NH}_{4}, 2 \mathrm{M}+\mathrm{Na}\end{array}$ & $\mathrm{C}_{55} \mathrm{H}_{100} \mathrm{O}_{6}$ & Glycerolipids & 0.1117 \\
\hline 6 & R4 & 782.567 & 4.23 & 4.39906 & $\downarrow$ & $\mathrm{PA}(17: 2 / 22: 1)$ & $\mathrm{M}+\mathrm{ACN}+\mathrm{H}$ & $\mathrm{C}_{42} \mathrm{H}_{77} \mathrm{O}_{8} \mathrm{P}$ & Glycerophospholipids & $0.0074^{* *}$ \\
\hline 7 & - & 903.739 & 14.55 & 3.8738 & $\downarrow$ & $\begin{array}{l}\text { TG(16:1/18:1/ } \\
\text { 20:3)[iso6] }\end{array}$ & $\mathrm{M}+\mathrm{Na}, \mathrm{M}+\mathrm{K}$ & $\mathrm{C}_{57} \mathrm{H}_{100} \mathrm{O}_{6}$ & Glycerolipids & $0.0017^{* *}$ \\
\hline 8 & R5 & 900.801 & 14.84 & 3.45942 & $\uparrow$ & $\begin{array}{l}\text { TG(16:0/18:1/ } \\
\text { 20:3)[iso6] }\end{array}$ & $\mathrm{M}+\mathrm{NH}_{4}, \mathrm{M}+\mathrm{Na}$ & $\mathrm{C}_{57} \mathrm{H}_{102} \mathrm{O}_{6}$ & Glycerolipids & $0.0174^{*}$ \\
\hline 9 & R6 & 902.816 & 15.11 & 3.44338 & $\uparrow$ & $\begin{array}{l}\text { TG(16:0/18:3/ } \\
\text { 20:0)[iso6] }\end{array}$ & $\mathrm{M}+\mathrm{NH}_{4}, \mathrm{M}+\mathrm{Na}, 2 \mathrm{M}+\mathrm{Na}$ & $\mathrm{C}_{57} \mathrm{H}_{104} \mathrm{O}_{6}$ & Glycerolipids & $0.0035^{* *}$ \\
\hline 10 & R7 & 666.618 & 14.64 & 3.39521 & $\uparrow$ & $\begin{array}{l}\text { Stigmasteryl } \\
\text { ester(16:1) }\end{array}$ & $\begin{array}{l}\mathrm{M}+\mathrm{H}, \mathrm{M}+\mathrm{NH}_{4}, \mathrm{M}+\mathrm{K}, 2 \mathrm{M}+ \\
\mathrm{NH}_{4}, 2 \mathrm{M}+\mathrm{Na}\end{array}$ & $\mathrm{C}_{45} \mathrm{H}_{76} \mathrm{O}_{2}$ & Stero lipids & $\begin{array}{l}7.79 \mathrm{E}- \\
08^{* * *}\end{array}$ \\
\hline 11 & R8 & 834.598 & 5.99 & 3.14588 & $\downarrow$ & $\operatorname{PS}(20: 0 / 19: 0)$ & $\begin{array}{l}\mathrm{M}+\mathrm{H}-2 \mathrm{H}_{2} \mathrm{O}, \mathrm{M}+\mathrm{H}, \mathrm{M}+ \\
\mathrm{Na}, 2 \mathrm{M}+\mathrm{H}, 2 \mathrm{M}+\mathrm{Na}\end{array}$ & $\mathrm{C}_{45} \mathrm{H}_{88} \mathrm{NO}_{10} \mathrm{P}$ & Glycerophospholipids & $0.0019^{* *}$ \\
\hline 12 & R9 & 369.352 & 14.65 & 3.00377 & $\uparrow$ & $\begin{array}{l}\text { (E,E)-3,7,11- } \\
\text { Trimethyl-2, } \\
\text { 6,10-dodecatrienyl } \\
\text { dodecanoate }\end{array}$ & $\mathrm{M}+\mathrm{H}-2 \mathrm{H}_{2} \mathrm{O}$ & $\mathrm{C}_{27} \mathrm{H}_{48} \mathrm{O}_{2}$ & Fatty acids & $\begin{array}{l}7.31 \mathrm{E}- \\
08^{* * * *}\end{array}$ \\
\hline 13 & R10 & 577.519 & 15.08 & 2.65073 & $\uparrow$ & $\mathrm{DG}(16: 0 / 18: 1 / 0: 0)$ & $\mathrm{M}+\mathrm{H}-\mathrm{H}_{2} \mathrm{O}$ & $\mathrm{C}_{37} \mathrm{H}_{70} \mathrm{O}_{5}$ & Glycerolipids & $08^{* * *}$ \\
\hline 14 & R11 & 848.769 & 14.77 & 2.49731 & $\uparrow$ & $\begin{array}{l}\text { TG(16:0/16:1/ } \\
\text { 18:1)[iso6] }\end{array}$ & $\mathrm{M}+\mathrm{H}, \mathrm{M}+\mathrm{NH}_{4}, \mathrm{M}+\mathrm{Na}, \mathrm{M}+\mathrm{K}$ & $\mathrm{C}_{53} \mathrm{H}_{98} \mathrm{O}_{6}$ & Glycerolipids & $0.0174^{*}$ \\
\hline 15 & $\mathrm{R} 12$ & 780.552 & 4.07 & 2.47828 & $\downarrow$ & $\operatorname{PE}(20: 0 / 17: 2)$ & $\mathrm{M}+\mathrm{Na}$ & $\mathrm{C}_{42} \mathrm{H}_{80} \mathrm{NO}_{8} \mathrm{P}$ & Glycerophospholipids & $0.0232^{*}$ \\
\hline 16 & R13 & 603.535 & 15.07 & 2.44092 & $\uparrow$ & $\mathrm{DG}(\mathrm{O}-16: 0 / 18: 1)$ & $\mathrm{M}+\mathrm{Na}$ & $\mathrm{C}_{37} \mathrm{H}_{72} \mathrm{O}_{4}$ & Glycerolipids & $08^{* * * *}$ \\
\hline 17 & - & 758.569 & 3.96 & 2.34541 & $\downarrow$ & $P C(16: 0 / 18: 2)$ & $\mathrm{M}+\mathrm{H}$ & $\mathrm{C}_{42} \mathrm{H}_{80} \mathrm{NO}_{8} \mathrm{P}$ & Glycerophospholipids & 0.5407 \\
\hline 18 & R14 & 701.558 & 3.58 & 2.32011 & $\downarrow$ & $\operatorname{SM}(d 16: 1 / 18: 1)$ & $\begin{array}{l}\mathrm{M}+\mathrm{H}, \mathrm{M}+\mathrm{Na}, \mathrm{M}+\mathrm{K}, 2 \mathrm{M}+ \\
\mathrm{H}, 2 \mathrm{M}+\mathrm{Na}\end{array}$ & $\mathrm{C}_{39} \mathrm{H}_{77} \mathrm{~N}_{2} \mathrm{O}_{6} \mathrm{P}$ & Sphingolipids & $0.0006^{* * *}$ \\
\hline 19 & R15 & 496.339 & 0.75 & 2.29702 & $\downarrow$ & $P C(0: 0 / 16: 0)$ & $\begin{array}{l}\mathrm{M}+\mathrm{H}-\mathrm{H}_{2} \mathrm{O}, \mathrm{M}+\mathrm{H}, \mathrm{M}+\mathrm{Na}, \mathrm{M}+ \\
\mathrm{K}, 2 \mathrm{M}+\mathrm{K}\end{array}$ & $\mathrm{C}_{24} \mathrm{H}_{50} \mathrm{NO}_{7} \mathrm{P}$ & Glycerophospholipids & $0.0049^{* *}$ \\
\hline 20 & R16 & 369.352 & 15.00 & 2.27907 & $\uparrow$ & 3-Deoxyvitamin D3 & $\mathrm{M}+\mathrm{H}, \mathrm{M}+\mathrm{NH}_{4,2} \mathrm{M}+\mathrm{H}$ & $\mathrm{C}_{27} \mathrm{H}_{44}$ & Stero lipids & $\begin{array}{l}7.31 \mathrm{E}- \\
08^{* * * *}\end{array}$ \\
\hline 21 & - & 870.753 & 14.27 & 2.16619 & $\downarrow$ & $\begin{array}{l}\text { TG(14:1/16:0/ } \\
\text { 22:4)[iso6] }\end{array}$ & $\mathrm{M}+\mathrm{NH}_{4}, \mathrm{M}+\mathrm{Na}, \mathrm{M}+\mathrm{K}$ & $\mathrm{C}_{55} \mathrm{H}_{96} \mathrm{O}_{6}$ & Glycerolipids & 0.2829 \\
\hline 22 & R17 & 925.796 & 14.55 & 2.1151 & $\uparrow$ & $\begin{array}{l}\text { TG(18:4/20:3/ } \\
\text { 22:0)[iso6] }\end{array}$ & $\mathrm{M}+\mathrm{H}-2 \mathrm{H}_{2} \mathrm{O}$ & $\mathrm{C}_{63} \mathrm{H}_{108} \mathrm{O}_{6}$ & Glycerolipids & $\begin{array}{l}7.86 \mathrm{E}- \\
08^{* * * *}\end{array}$ \\
\hline 23 & R18 & 801.682 & 10.78 & 2.06029 & $\downarrow$ & $\mathrm{SM}(\mathrm{d} 17: 1 / 24: 0)$ & $\mathrm{M}+\mathrm{H}, \mathrm{M}+\mathrm{Na}$ & $\mathrm{C}_{46} \mathrm{H}_{93} \mathrm{~N}_{2} \mathrm{O}_{6} \mathrm{P}$ & Sphingolipids & $\begin{array}{l}1.85 \mathrm{E}- \\
05^{* * *}\end{array}$ \\
\hline 24 & - & 899.709 & 13.99 & 1.99376 & $\downarrow$ & $\begin{array}{l}\text { TG(16:1/18:2/ } \\
\text { 20:4)[iso6] }\end{array}$ & $\mathrm{M}+\mathrm{NH} 4, \mathrm{M}+\mathrm{Na}, \mathrm{M}+\mathrm{K}$ & $\mathrm{C}_{57} \mathrm{H}_{96} \mathrm{O}_{6}$ & Glycerolipids & 0.1458 \\
\hline 25 & R19 & 546.352 & 1.24 & 1.88084 & $\downarrow$ & $\operatorname{PS}(22: 0 / 0: 0)$ & $\mathrm{M}+\mathrm{H}-2 \mathrm{H}_{2} \mathrm{O}$ & $\mathrm{C}_{28} \mathrm{H}_{56} \mathrm{NO}_{9} \mathrm{P}$ & Glycerophospholipids & $0.0249^{*}$ \\
\hline 26 & - & 873.693 & 13.97 & 1.87046 & $\downarrow$ & $\begin{array}{l}\text { TG(15:1/17:1/ } \\
\text { 20:4)[iso6] }\end{array}$ & $\mathrm{M}+\mathrm{Na}, \mathrm{M}+\mathrm{K}$ & $\mathrm{C}_{55} \mathrm{H}_{94} \mathrm{O}_{6}$ & Glycerolipids & 0.1245 \\
\hline 27 & - & 834.598 & 5.14 & 1.83438 & $\downarrow$ & $P C(22: 4 / 18: 2)$ & $\mathrm{M}+\mathrm{H}, \mathrm{M}+\mathrm{Na}, 2 \mathrm{M}+\mathrm{Na}$ & $\mathrm{C}_{48} \mathrm{H}_{84} \mathrm{NO}_{8} \mathrm{P}$ & Glycerophospholipids & 0.4226 \\
\hline
\end{tabular}


Table 2 List of assigned statistically significantly lipid molecules and change trend of biomarkers on plasma lipid profile in positive ion mode after comparison of IPF patient samples to control samples (Continued)

\begin{tabular}{|c|c|c|c|c|c|c|c|c|c|c|}
\hline No. & Code & $\begin{array}{l}\text { Calc m/ } \\
z\end{array}$ & $t_{R}$ & VIP & Trend $^{a}$ & Accepted Description & Lipidlon & Element & Lipid class & $P$-Value \\
\hline 28 & R20 & 832.580 & 5.49 & 1.81376 & $\downarrow$ & $P C(20: 2 / 18: 2)$ & $\mathrm{M}+\mathrm{Na}, 2 \mathrm{M}+\mathrm{Na}$ & $\mathrm{C}_{46} \mathrm{H}_{84} \mathrm{NO}_{8} \mathrm{P}$ & Glycerophospholipids & $\begin{array}{l}1.22 \mathrm{E}- \\
06^{* * *}\end{array}$ \\
\hline 29 & - & 931.771 & 15.01 & 1.81008 & $\downarrow$ & $\begin{array}{l}\text { TG(17:0/17:0/ } \\
\text { 22:5)[iso3] }\end{array}$ & $\mathrm{M}+\mathrm{Na}$ & $\mathrm{C}_{59} \mathrm{H}_{104} \mathrm{O}_{6}$ & Glycerolipids & 0.5582 \\
\hline 30 & - & 786.601 & 4.84 & 1.76508 & $\downarrow$ & $P C(18: 1 / 18: 1)$ & $\begin{array}{l}\mathrm{M}+\mathrm{H}-\mathrm{H}_{2} \mathrm{O}, \mathrm{M}+\mathrm{H} \\
\mathrm{M}+\mathrm{K}\end{array}$ & $\mathrm{C}_{44} \mathrm{H}_{84} \mathrm{NO}_{8} \mathrm{P}$ & Glycerophospholipids & 0.9026 \\
\hline 31 & - & 1564.121 & 2.72 & 1.68796 & $\downarrow$ & $P C(22: 4 / 14: 0)$ & $\begin{array}{l}\mathrm{M}+\mathrm{NH}_{4}, \mathrm{M}+\mathrm{K} \\
2 \mathrm{M}+\mathrm{H}\end{array}$ & $\mathrm{C}_{44} \mathrm{H}_{80} \mathrm{NO}_{8} \mathrm{P}$ & Glycerophospholipids & 0.2314 \\
\hline 32 & R21 & 603.532 & 14.82 & 1.64593 & $\uparrow$ & $\begin{array}{l}\text { DG(18:0/18:2/0:0) } \\
\text { [iso2] }\end{array}$ & $\mathrm{M}+\mathrm{H}-\mathrm{H}_{2} \mathrm{O}$ & $\mathrm{C}_{39} \mathrm{H}_{72} \mathrm{O}_{5}$ & Glycerolipids & $\begin{array}{l}7.53 \mathrm{E}- \\
08^{* * * *}\end{array}$ \\
\hline 33 & R22 & 575.503 & 14.54 & 1.62251 & $\uparrow$ & $\begin{array}{l}\text { DG(16:0/18:2/0:0) } \\
\text { [iso2] }\end{array}$ & $\mathrm{M}+\mathrm{H}-\mathrm{H}_{2} \mathrm{O}$ & $\mathrm{C}_{37} \mathrm{H}_{68} \mathrm{O}_{5}$ & Glycerolipids & $\begin{array}{l}2.58 \mathrm{E}- \\
07^{* * * *}\end{array}$ \\
\hline 34 & - & 812.614 & 6.86 & 1.61868 & $\downarrow$ & PS(O-20:0/20:2) & $\begin{array}{l}\mathrm{M}+\mathrm{H}-\mathrm{H}_{2} \mathrm{O} \\
\mathrm{M}+\mathrm{H}\end{array}$ & $\mathrm{C}_{46} \mathrm{H}_{88} \mathrm{NO}_{9} \mathrm{P}$ & Glycerophospholipids & 0.0893 \\
\hline 35 & - & 849.693 & 14.21 & 1.61511 & $\downarrow$ & $\begin{array}{l}\text { TG(14:1/18:1/ } \\
\text { 18:2)[iso6] }\end{array}$ & $\begin{array}{l}\mathrm{M}+\mathrm{NH}_{4}, \mathrm{M}+\mathrm{Na} \\
\mathrm{M}+\mathrm{K}\end{array}$ & $\mathrm{C}_{53} \mathrm{H}_{94} \mathrm{O}_{6}$ & Glycerolipids & 0.2829 \\
\hline 36 & - & 904.831 & 15.36 & 1.53098 & $\uparrow$ & $\begin{array}{l}\text { TG(17:0/18:1/ } \\
\text { 19:1)[iso6] }\end{array}$ & $\begin{array}{l}\mathrm{M}+\mathrm{NH}_{4}, \mathrm{M}+\mathrm{Na} \\
\mathrm{M}+\mathrm{K}, 2 \mathrm{M}+\mathrm{Na}\end{array}$ & $\mathrm{C}_{57} \mathrm{H}_{106} \mathrm{O}_{6}$ & Glycerolipids & 0.1615 \\
\hline 37 & R23 & 828.551 & 5.01 & 1.5305 & $\downarrow$ & PS(P-18:0/19:0) & $\mathrm{M}+\mathrm{H}-\mathrm{H}_{2} \mathrm{O}, \mathrm{M}+\mathrm{K}$ & $\mathrm{C}_{43} \mathrm{H}_{84} \mathrm{NO}_{9} \mathrm{P}$ & Glycerophospholipids & $0.0428^{*}$ \\
\hline 38 & R24 & 599.504 & 14.54 & 1.49112 & $\uparrow$ & $\begin{array}{l}\text { DG(18:1/18:3/0:0) } \\
\text { [iso2] }\end{array}$ & $\mathrm{M}+\mathrm{H}-\mathrm{H}_{2} \mathrm{O}, \mathrm{M}+\mathrm{H}$ & $\mathrm{C}_{39} \mathrm{H}_{68} \mathrm{O}_{5}$ & Glycerolipids & $\begin{array}{l}1.21 \mathrm{E}- \\
07^{* * * *}\end{array}$ \\
\hline 39 & - & 901.725 & 13.34 & 1.48742 & $\downarrow$ & $\begin{array}{l}\text { TG(16:0/16:0/ } \\
\text { 22:6)[iso3] }\end{array}$ & $\mathrm{M}+\mathrm{NH}_{4}, \mathrm{M}+\mathrm{Na}$ & $\mathrm{C}_{57} \mathrm{H}_{98} \mathrm{O}_{6}$ & Glycerolipids & 0.7340 \\
\hline 40 & R25 & 955.759 & 13.01 & 1.48372 & $\downarrow$ & $\begin{array}{l}\text { TG(19:1/22:6/ } \\
\text { 22:6)[iso3] }\end{array}$ & $\mathrm{M}+\mathrm{H}-2 \mathrm{H}_{2} \mathrm{O}$ & $\mathrm{C}_{66} \mathrm{H}_{102} \mathrm{O}_{6}$ & Glycerolipids & $\begin{array}{l}3.56 \mathrm{E}- \\
06^{* * * *}\end{array}$ \\
\hline 41 & - & 925.724 & 14.16 & 1.46671 & $\downarrow$ & $\begin{array}{l}\text { TG(12:0/22:2/ } \\
\text { 22:6)[iso6] }\end{array}$ & $\begin{array}{l}\mathrm{M}+\mathrm{NH}_{4}, \mathrm{M}+\mathrm{Na} \\
\mathrm{M}+\mathrm{K}\end{array}$ & $\mathrm{C}_{59} \mathrm{H}_{98} \mathrm{O}_{6}$ & Glycerolipids & 0.1458 \\
\hline 42 & - & 879.739 & 13.99 & 1.38113 & $\downarrow$ & $\begin{array}{l}\text { GalCer(d18:1/ } \\
\text { 26:1(17Z)) }\end{array}$ & $\mathrm{M}+\mathrm{ACN}+\mathrm{H}$ & $\mathrm{C}_{50} \mathrm{H}_{95} \mathrm{NO}_{8}$ & Sphingolipids & 0.7340 \\
\hline 43 & R26 & 601.518 & 14.53 & 1.36641 & $\uparrow$ & $\begin{array}{l}\text { DG(18:1/18:2/0:0) } \\
\text { [iso2] }\end{array}$ & $\mathrm{M}+\mathrm{H}-\mathrm{H}_{2} \mathrm{O}$ & $\mathrm{C}_{39} \mathrm{H}_{70} \mathrm{O}_{5}$ & Glycerolipids & $\begin{array}{l}8.04 \mathrm{E}- \\
07^{* * *}\end{array}$ \\
\hline 44 & - & 810.599 & 4.57 & 1.32667 & $\downarrow$ & $P C(18: 0 / 18: 1)$ & $\mathrm{M}+\mathrm{Na}$ & $\mathrm{C}_{44} \mathrm{H}_{86} \mathrm{NO}_{8} \mathrm{P}$ & Glycerophospholipids & 0.1534 \\
\hline 45 & R27 & 759.637 & 6.96 & 1.30466 & $\downarrow$ & SM(d18:1/20:0) & $\mathrm{M}+\mathrm{H}, \mathrm{M}+\mathrm{Na}, 2 \mathrm{M}+\mathrm{H}$ & $\mathrm{C}_{43} \mathrm{H}_{87} \mathrm{~N}_{2} \mathrm{O}_{6} \mathrm{P}$ & Sphingolipids & $0.0139^{*}$ \\
\hline 46 & - & 524.371 & 1.10 & 1.29548 & $\downarrow$ & $P C(18: 0 / 0: 0)$ & $\mathrm{M}+\mathrm{H}, \mathrm{M}+\mathrm{K}$ & $\mathrm{C}_{26} \mathrm{H}_{54} \mathrm{NO}_{7} \mathrm{P}$ & Glycerophospholipids & 0.2479 \\
\hline 47 & R28 & 830.566 & 3.80 & 1.25023 & $\downarrow$ & PC(22:6/18:2) & $\mathrm{M}+\mathrm{H}, \mathrm{M}+\mathrm{Na}$ & $\mathrm{C}_{48} \mathrm{H}_{80} \mathrm{NO}_{8} \mathrm{P}$ & Glycerophospholipids & $0.0038^{* *}$ \\
\hline 48 & R29 & 1620.182 & 4.50 & 1.22041 & $\downarrow$ & PI-Cer(d20:0/16:0) & $\begin{array}{l}\mathrm{M}+\mathrm{H}-2 \mathrm{H}_{2} \mathrm{O}, \mathrm{M}+\mathrm{K} \\
2 \mathrm{M}+\mathrm{H}\end{array}$ & $\mathrm{C}_{42} \mathrm{H}_{84} \mathrm{NO}_{11} \mathrm{P}$ & Glycerophospholipids & $\begin{array}{l}7.90 \mathrm{E}- \\
08^{* * *}\end{array}$ \\
\hline 49 & - & 828.551 & 4.04 & 1.20343 & $\downarrow$ & $\operatorname{PE}(22: 6 / 19: 0)$ & $\mathrm{M}+\mathrm{Na}$ & $\mathrm{C}_{46} \mathrm{H}_{80} \mathrm{NO}_{8} \mathrm{P}$ & Glycerophospholipids & 0.2060 \\
\hline 50 & R30 & 948.800 & 14.59 & 1.20004 & $\uparrow$ & $\begin{array}{l}\text { TG(18:1/20:3/ } \\
\text { 20:4)[iso6] }\end{array}$ & $\mathrm{M}+\mathrm{NH}_{4}$ & $\mathrm{C}_{61} \mathrm{H}_{102} \mathrm{O}_{6}$ & Glycerolipids & $\begin{array}{l}7.90 \mathrm{E}- \\
08^{* * * *}\end{array}$ \\
\hline 51 & R31 & 794.602 & 4.86 & 1.19345 & $\downarrow$ & PC(20:3/P-18:1) & $\begin{array}{l}\mathrm{M}+\mathrm{H}, \mathrm{M}+\mathrm{Na} \\
2 \mathrm{M}+\mathrm{H}\end{array}$ & $\mathrm{C}_{46} \mathrm{H}_{84} \mathrm{NO}_{7} \mathrm{P}$ & Glycerophospholipids & $\begin{array}{l}1.93 \mathrm{E}- \\
07^{* * * *}\end{array}$ \\
\hline 52 & R32 & 790.569 & 5.79 & 1.18604 & $\downarrow$ & PA(20:5/20:1) & $\begin{array}{l}\mathrm{M}+\mathrm{H}-\mathrm{H}_{2} \mathrm{O} \\
\mathrm{M}+\mathrm{ACN}+\mathrm{H}\end{array}$ & $\mathrm{C}_{43} \mathrm{H}_{73} \mathrm{O}_{8} \mathrm{P}$ & Glycerophospholipids & $0.0012^{* *}$ \\
\hline 53 & - & 881.754 & 14.25 & 1.18421 & $\downarrow$ & $\begin{array}{l}\text { TG(15:0/18:1/ } \\
\text { 19:1)[iso6] }\end{array}$ & $\mathrm{M}+\mathrm{Na}$ & $\mathrm{C}_{55} \mathrm{H}_{102} \mathrm{O}_{6}$ & Glycerolipids & 0.2829 \\
\hline 54 & - & 1494.138 & 6.25 & 1.17806 & $\downarrow$ & $\begin{array}{l}C L\left(11^{\prime}-[20: 0 / 18: 0], 3^{\prime}-\right. \\
[18: 0 / 18: 0])\end{array}$ & $\mathrm{M}+\mathrm{H}$ & $\mathrm{C}_{83} \mathrm{H}_{162} \mathrm{O}_{17} \mathrm{P}_{2}$ & Glycerophospholipids & 0.5751 \\
\hline 55 & R33 & 851.708 & 13.63 & 1.14176 & $\downarrow$ & 20:1-Glc-Sitosterol & $\mathrm{M}+\mathrm{H}-\mathrm{H}_{2} \mathrm{O}$ & $\mathrm{C}_{55} \mathrm{H}_{96} \mathrm{O}_{7}$ & Stero lipids & $0.0161^{*}$ \\
\hline 56 & R34 & 897.696 & 13.73 & 1.10216 & $\downarrow$ & & $\mathrm{M}+\mathrm{H}$ & $\mathrm{C}_{59} \mathrm{H}_{92} \mathrm{O}_{6}$ & Glycerolipids & $0.0014^{* *}$ \\
\hline
\end{tabular}


Table 2 List of assigned statistically significantly lipid molecules and change trend of biomarkers on plasma lipid profile in positive ion mode after comparison of IPF patient samples to control samples (Continued)

\begin{tabular}{|c|c|c|c|c|c|c|c|c|c|c|}
\hline No. & Code & $\begin{array}{l}\text { Calc m/ } \\
\mathrm{z}\end{array}$ & $t_{R}$ & VIP & Trend $^{a}$ & Accepted Description & Lipidlon & Element & Lipid class & $P$-Value \\
\hline & & & & & & $\begin{array}{l}\text { TG(18:3/18:3/ } \\
\text { 20:5)[iso3] }\end{array}$ & & & & \\
\hline 57 & - & 542.321 & 0.60 & 1.09805 & $\downarrow$ & $P C(20: 5 / 0: 0)$ & $\mathrm{M}+\mathrm{H}$ & $\mathrm{C}_{28} \mathrm{H}_{48} \mathrm{NO}_{7} \mathrm{P}$ & Glycerophospholipids & 0.3318 \\
\hline 58 & - & 1576.219 & 8.50 & 1.05263 & $\downarrow$ & $P C(17: 0 / 19: 1)$ & $M+K, 2 M+H$ & $\mathrm{C}_{44} \mathrm{H}_{86} \mathrm{NO}_{8} \mathrm{P}$ & Glycerophospholipids & 0.2471 \\
\hline 59 & - & 951.740 & 14.31 & 1.04393 & $\downarrow$ & $\begin{array}{l}\text { TG(18:4/20:0/ } \\
\text { 20:5)[iso6] }\end{array}$ & $\mathrm{M}+\mathrm{Na}, \mathrm{M}+\mathrm{K}$ & $\mathrm{C}_{61} \mathrm{H}_{100} \mathrm{O}_{6}$ & Glycerolipids & 0.3918 \\
\hline 60 & - & 896.768 & 14.27 & 1.01614 & $\downarrow$ & $\begin{array}{l}\text { TG(18:1/18:2/ } \\
\text { 18:3)[iso6] }\end{array}$ & $\mathrm{M}+\mathrm{NH}_{4}, \mathrm{M}+\mathrm{Na}$ & $\mathrm{C}_{57} \mathrm{H}_{98} \mathrm{O}_{6}$ & Glycerolipids & 0.9892 \\
\hline 61 & R35 & 920.768 & 14.30 & 1.01291 & $\uparrow$ & $\begin{array}{l}\text { TG(16:0/20:3/ } \\
\text { 20:5)[iso6] }\end{array}$ & $\mathrm{M}+\mathrm{NH}_{4}, \mathrm{M}+\mathrm{K}$ & $\mathrm{C}_{59} \mathrm{H}_{98} \mathrm{O}_{6}$ & Glycerolipids & $\begin{array}{l}1.40 \mathrm{E}- \\
06^{* * * *}\end{array}$ \\
\hline 62 & - & 905.754 & 13.68 & 1.00186 & $\downarrow$ & $\begin{array}{l}\text { TG(12:0/20:1/ } \\
\text { 22:3)[iso6] }\end{array}$ & $\mathrm{M}+\mathrm{Na}$ & $\mathrm{C}_{57} \mathrm{H}_{102} \mathrm{O}_{6}$ & Glycerolipids & 0.8811 \\
\hline
\end{tabular}

${ }^{a}$ Change trend of lipids on IPF patients vs controls. The levels of potential lipid biomarkers labeled with $(\uparrow)$ and $(\downarrow)$ represent up-regulation and down-regulation, respectively. The variable importance for projection (VIP) statistics and T-test were used applied to select significant variables leading to group separation. VIP value larger than 1.0 were considered statistically significant and significant differences in statistics were also defined by $\mathrm{P}$ values of $<0.05(*),<0.01(* *)$, and $<0.001\left(^{* * *}\right)$

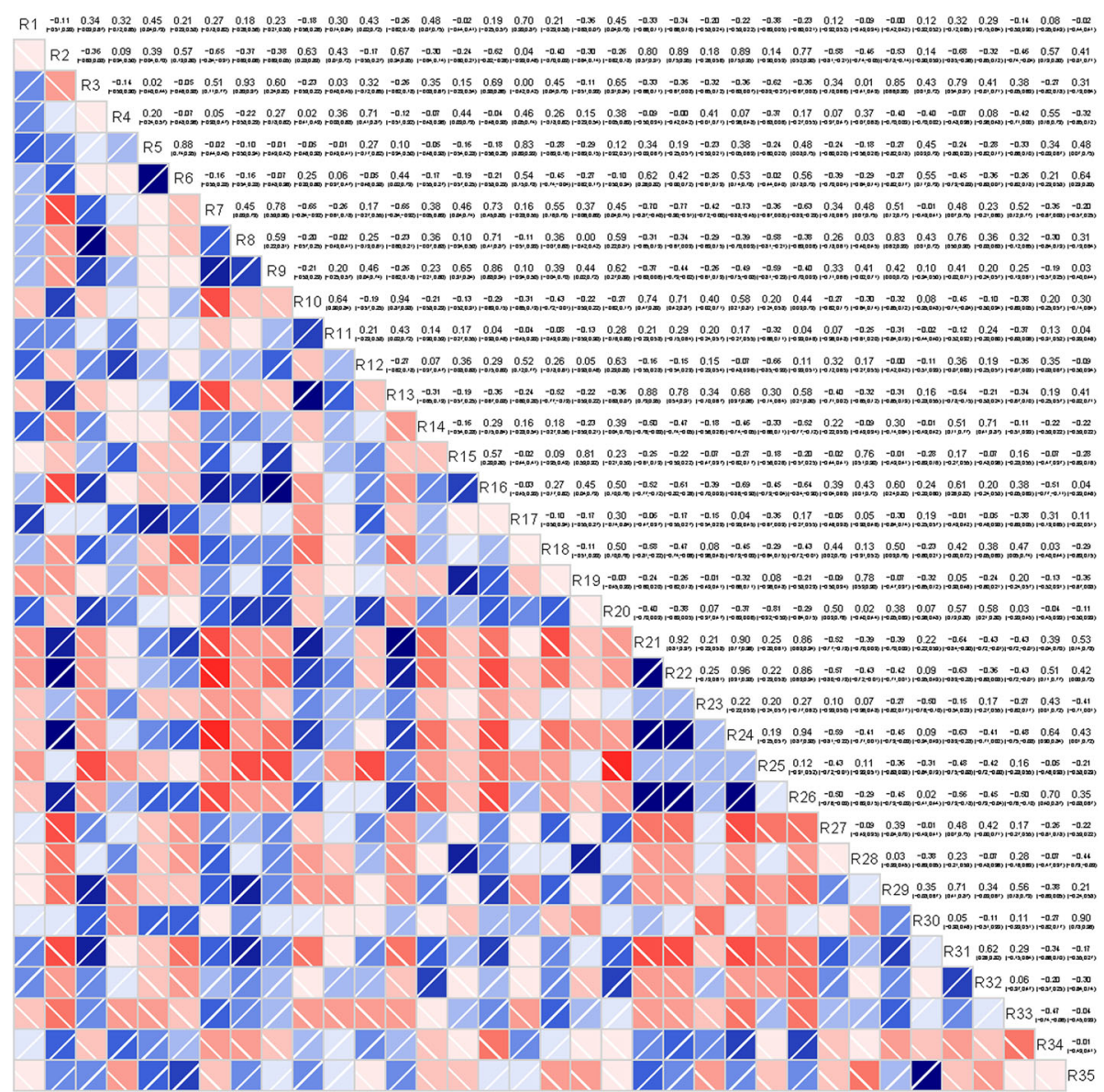

Fig. 2 Correlation analysis of the 35 pre-selected discriminating lipids in IPF patients and controls. R1 to R35 represents the corresponding pre-selected discriminating lipids as shown in Table 2. Red and blue represent a negative and positive correlation, respectively. The color depth represents the degree of correlation: the deeper color indicates higher correlation 
IPF objects and controls, the receiver operating characteristic (ROC) curve was applied according to the results for the area under the curve (AUC) and sensitivity/specificity at the best cut-off points (Fig. 3). The AUC values of these molecules showed significant differences in the discovery set. 6 (R7, R9, R13, R16, R17 and R21) of them showed higher sensitivity/specificity for identifying IPF objects from controls (Fig. 3). This result have demonstrated that each of the sixmolecules may be used as potential biomarkers in diagnosing IPF disease in the future.

Gender, smoking, and disease status-associated differences in the lipid levels of six-promising biomarkers

To demonstrate whether gender, smoking history, and disease stage have influence on selecting useful future
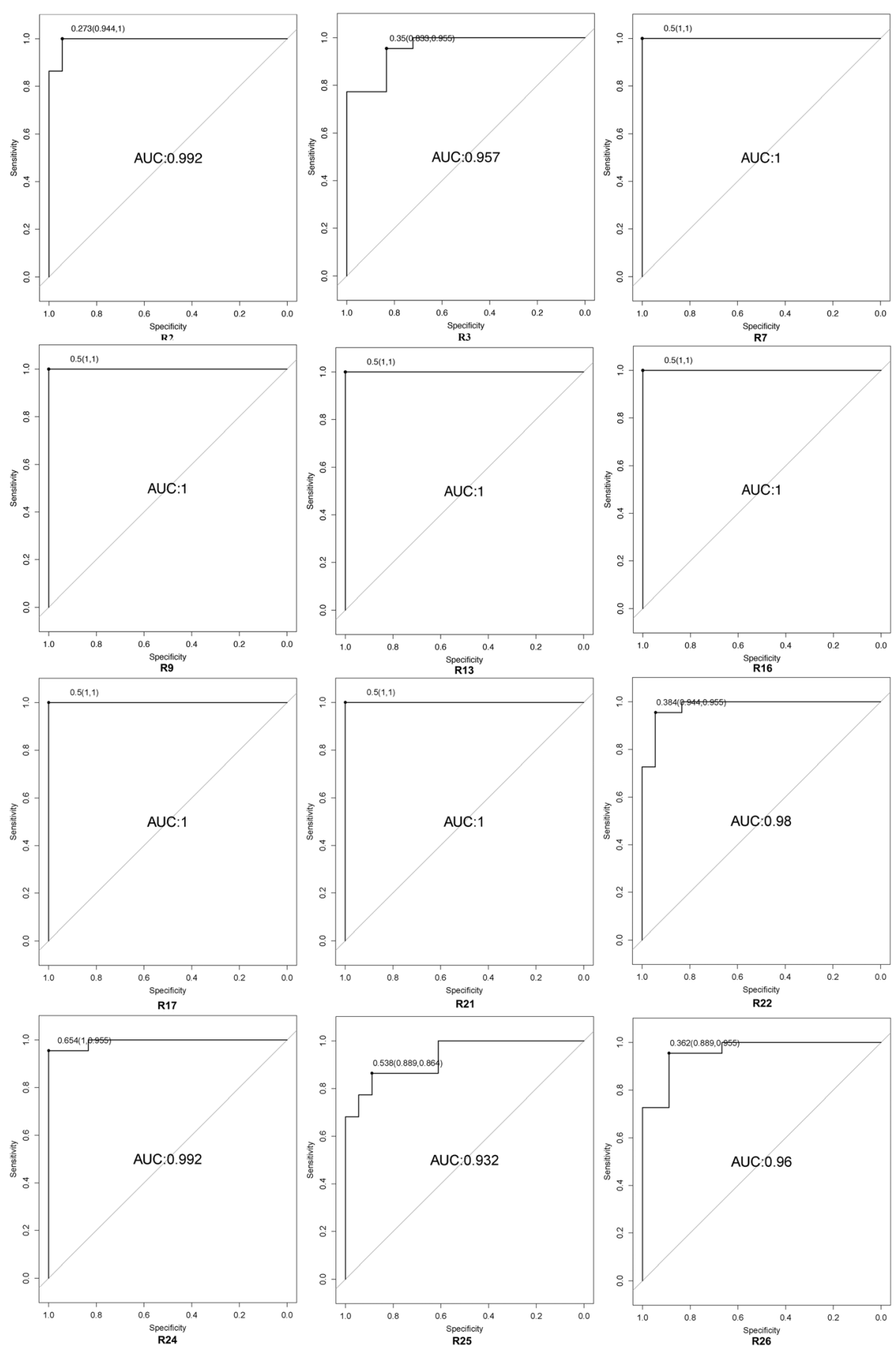

Fig. 3 ROC curves analysis of 12 lipid metabolite for discriminating IPF objects from controls 
biomarkers, 6 lipid levels in serums from IPF objects and control were further studied. Table 3 summarises the significance of all 6 biomarkers with regards to gender, smoking history, and disease stage-associated differences. None of these 6 was found to have significant differences related to these parameters mentioned above.

\section{Discussion}

IPF is a kind of chronic and progressive disease with low survival rate, and remains to be a clinical challenge. It is regarded as a fetal disease due to its poor prognosis and a low median survival of only $3 \sim 5$ years after diagnosis. At the moment, only clinical data are available for diagnosis to researchers and clinicians, which is of limited value as they do not reflect the precise pathological mechanisms underlying IPF. To better elucidate disease mechanism and make early diagnosis of IPF, identification of molecular biomarkers with high diagnostic value is paramount. In recent publications, a number of biomarkers have been hypothesized to be present in serum used as potential prognostic or diagnostic tools. The main protein biomarkers associated with cell dysfunction are the Krebs con den lungen-6 (KL-6) antigen and the surfactant protein A and protein D (SP-A and SP-D) [39]. Biomarkers found in IPF involved in fibrogenesis are matrix metalloproteinases-1 and -7 (MMP-1 and MMP-7), which play a role in the breakdown and remodeling of extracellular matrix components [40]. However, no lipid biomarkers have yet been studied for IPF. This work is the first comprehensive investigation into the potential lipid biomarkers for IPF using UPSL-QTOF-MS/MS.

Lipids is the fundamental component of cellular membranes, also exert several essential and critical roles in cellular functions including energy storage, signal transduction, formation of membrane bilayer and cellular barriers. The metabolism of lipid is indicated in numerous human diseases, such as Alzheimer's disease, diabetes, obesity, atherosclerosis and several types of respiratory diseases. There are eight major categories of lipid types based on their structures: fatty acid, glycerolipid, saccharolipid, polyketide, sphingolipid, sterol lipids,

Table 3 Differences in the lipid levels of eight-promising biomarkers under the influence of gender, smoking, and disease status. The statistical significance was determined by Mann-Whitney U-test

\begin{tabular}{llll}
\hline No. & Gender/P-value & Smoking/P-value & Disease status/P-value \\
\hline R7 & 0.92 & 0.92 & 0.65 \\
R9 & 0.51 & 0.51 & 0.91 \\
R13 & 0.81 & 0.16 & 0.17 \\
R16 & 0.40 & 0.40 & 0.84 \\
R17 & 0.85 & 0.43 & 0.30 \\
R21 & 0.96 & 0.17 & 0.15 \\
\hline
\end{tabular}

prenol lipid and glycerophospholipid. Although abnormal lipid metabolism has been shown to result in cystic fibrosis and lung injury published by Ollero's and Goss' research groups, respectively [41, 42], its potential role in pathology of IPF remains unclear.

In this study, analysis of lipid profile from 22 IPF patients and 18 control subjects revealed the characterization of lipid composition. The glycerophospholipids (GPs) appears to be the important biological molecules for the backbone of cellular membranes. Besides an integral component of biomembranes, GPs also seem to be a reservoir of a large amount of many bioactive mediators [43], which are produced by the reaction of phospholipases on GPs. GPs can be further divided into different categories including glycerophosphoglycerols (PGs), glycerophosphatidic acids (PAs), glycerophosphoinositols (PIs), glycerophosphoserines (PSs), glycerophosphoethanolamines (PEs) and cardiolipin (CLs).

In our study, 2 CLs, 2 PAs, 13 PCs, 2 PEs, 1 PIs, and 4 PSs were identified as unique lipids of IPF patients based on VIP scores (Table 2). The levels of all screened GPs was decreased in IPF patients compared to the control subjects. This phenomenon may be due to the fact that PG is a precursor of CL biosynthesis and PA is the critic substrate for biosynthesis of PI, PG, PE, and PC. A previous TLC-MALDI/TOF-based metabonomics study displayed significantly decreased plasma $\mathrm{PCs}$ in cystic fibrosis patients, including $\mathrm{PC}(\mathrm{P}-40: 1), \quad \mathrm{PC}(38: 6)$, $\mathrm{PC}(38: 5), \quad \mathrm{PC}(38: 4), \mathrm{PC}(38: 2), \mathrm{PC}(38: 0)$ and $\mathrm{PC}(36: 5)$ [44]. However, using electrospray ionization tandem mass spectrometry (ESI-MS/MS)-based metabonomics, Marien et al. showed an increase in non-small cell lung cancer of $\operatorname{PI}(38: 3), \operatorname{PI}(40: 3), \operatorname{PI}(38: 2), \operatorname{PS}(32: 0), \operatorname{PS}(36: 4)$, PS(36:1), PS(40:2), PS(38:1), PS(34:0), PS(40:1), PS(38:5), PS(34:2) and PS(38:4), compared to normal lung tissues [45]. Increasing evidence implicates that GPs biomarkers were several in agreement with abnormal GPs metabolism found in above mentioned lung disease and IPF model. This result indicated that GPs with top scores could be used as potential biomarkers for IPF.

Our study identified 159 glycerolipids in IPF patients and 30 out of these 159 have the capacity to differentiate IPF patients from control subjects (VIP $>1$, Table 2). Glycerolipids possess long chain fatty acids in ester linkage to the glycerol backbone and were classified into two major groups: diacylglycerol (DG) and triacylglycerol (TG), which are the most abundant lipids found in circulating plasma [46]. For example, all six DGs out of 30 glycerolipids showed similar tendencies to increase in the plasma from IPF patients with variable magnitudes. Interestingly, 9 out of 24 TGs displayed increased levels in IPF patients compared to control subjects. A previous study demonstrated that glycerolipids were significantly reduced in lung tissues of mice, which lacked the p53 oncogene [47]. This result indicates that the abnormal 
function of glycerolipid was associated with lung disease. Further investigation of the exact roles of glycerolipids in IPF patients would be beneficial.

We detected 47 sphingolipids (SLs) by UPSL-QTOF-MS/ MS in plasma samples and found that 4 out of them can distinguish the IPF patients from control subjects. 4 types of SLs (GalCerd18:1/26:1, d18:1/20:0, d16:1/18:1, d17:1/ 24:0) significantly decreased in plasma of patients with IPF. SLs are ubiquitous cellular membrane components that are implicated in multiple cellular processes including autophagy, apoptosis, differentiation and cell division [48]. Ceramide is generated from either sohingomyelin or de novo sphingolipids synthesis [49] and its upregulation has been found in chronic-obstructive pulmonary disease [50]. In contrast, our results showed decreased SLs serum level in IPF patient, which could be due to the differences between different respiratory diseases. In the future, we will compare SLs levels in serum and bronchoalveolar lavage fluids between respiratory diseases, such as COPD and pneumonia. Although it remains to be equivocal whether differences exist in SLs levels between IPF and other respiratory diseases, our data have provided a direction for future investigation into implications of these SLs in IPF patients.

Moreover, this study identified 46 kinds of serol lipids and 20 kinds of fatty acids. 3 kinds of 46 serol lipids and 1 kind of 20 fatty acids were considered as potential biomarkers. Fatty acids are the most important class of lipids and function as precursors of various bioactive lipid molecules. In our study, fatty acid (E,E)-3,7,11-Trimethyl2,6,10-dodecatrienyl dodecanoate was shown to be positively correlated with IPF and may distinguish IPF patients from control subjects. Three (3-Deoxyvitamin D3,16:1 Stigmasteryl ester, and 20:1-Glc-Sitosterol) out of 46 sterol lipids identified have been shown to correlate with the IPF. Other studies have shown that plasma cholesterol level was significantly increased in diet-induced hyperlipidomic rats [51], and cholesteryl ester apparent lipid molecular species have high sensitivity, specificity and accuracy in the diagnosis of prostate cancer [10]. Further validation of the specificity of these lipid molecules in IPF patients among respiratory disease would be desirable.

Furthermore, we investigated lipid molecules of 12 metabolites possessing close correlations with IPF disease. 6 of them showed higher sensitivity and specificity for identifying IPF patients from control subjects ROC analysis (Fig. 3). Previous study has demonstrated that age, gender, and smoking status can affect plasma lipid metabolite levels in healthy adults $[52,53]$. The impact of gender and smoking on 6 promising biomarker levels were determined in this study and no correlation was found (Table 3). Further validations on whether the 6 identified promising biomarkers has the increased ability to discriminate IPF objects from healthy controls or other respiratory disease are highly suggested.
For the analysis of IPF patients and biomarkers, the potential defect in this study was the small sample size. Another limitation is the lack of a longitudinal study, which made it impossible to observe the clinical impact of the present discovery as no treatment responses can be assessed. Although we have identified promising lipid biomarkers in this study, further validations are necessary to evaluate the specificity of the identified biomarkers for IPF diagnosis. Then, further longitudinal multicenter studies would contribute more to evaluate the real value of lipid biomarkers as diagnostic and prognostic tools. In addition, the lipid biomarkers identified in this study should be compared with known diagnostic and prognostic biomarkers in the future, such as KL-6, SP-A, SP-D. This will provide an insight into a better understanding of the diagnostic and prognostic utility of identified lipid biomarkers. Lastly, more specific lipidomics analyses of these lipid changes in IPF will probably help to better understand the IPF pathology and may contribute to future development of novel therapeutic targets.

\section{Conclusions}

In conclusions, our study has yielded important information regarding lipid metabolism in IPF patients and presents the first identification of promising potential biomarkers for the diagnosis of IPF. Our results demonstrate that individual lipid molecules have the ability to differentiate IPF from controls. Implications for future studies include validation of the accuracy of biomarkers to diagnose IPF and investigation in their IPF-specificity compared to other respiratory diseases, such as asthma, COPD, and infective pneumonia.

\section{Additional file}

\section{Additional file 1: Table S1. This file contains the plasma lipid profiles of 507 individual lipid species were determined with lipidomics from 40 plasma samples; 22 from IPF patients with 18 controls. (XLS 562 kb)}

\section{Abbreviations \\ CLs: Cardiolipin; IPF: Idiopathic pulmonary fibrosis; OPLS-DA: Orthogonal partial least squares discriminant analysis; PAs: Glycerophosphatidic acids; PCs: Glycerophosphocholines; PEs: Glycerophosphoethanolamines; PGs: Glycerophosphoglycerols; Pls: Glycerophosphoinositols; \\ PSs: Glycerophosphoserines; ROC: Receiver operating characteristic; UPLC-QTOF/MS: Ultraperformance liquid chromatography coupled with quadrupole time of flight mass spectrometry; VIP: Variable importance for projection}

\section{Acknowledgements}

Not applicable.

Funding

Not applicable.

Availability of data and materials

The datasets supporting the conclusions of this article is available in the results section. 


\section{Authors' contributions}

FY, ZSW, WJW and XYC made substantial contributions to conception and design, or acquisition of data, or analysis and interpretation of data. ZSW was involved in drafting the manuscript or revising it critically for important intellectual content. All authors read and approved the final manuscript.

\section{Ethics approval and consent to participate}

The clinical IPF samples included in this study were collected from the First hospital of Tsinghua University. All patient data were anonymous, so informed consent for participation was not required. The use of these samples was approved by the Institutional Review Board for human studies at the First Hospital of Tsinghua University.

\section{Consent for publication}

Not applicable.

\section{Competing interests}

The authors declare that they have no competing interests.

\section{Publisher's Note}

Springer Nature remains neutral with regard to jurisdictional claims in published maps and institutional affiliations.

\section{Author details}

'Department of Respiration, First Hospital of Tsinghua University, Beijing 100016, China. ${ }^{2}$ Division of Research and Education, First Hospital of Tsinghua University, Beijing 100016, China. ${ }^{3}$ Department of Neurology, The LongFu hospital of Beijing, Beijing 100010, China. ${ }^{4}$ Department of Gerontology, The First Hospital of ShanXi Medical University, Taiyuan, ShanXi 030001, China. ${ }^{5}$ Beijing Qiji Biotechnology Company, Beijing 100193, China.

Received: 6 January 2017 Accepted: 20 November 2017 Published online: 06 December 2017

\section{References}

1. Joo S, et al. Clinical results of sublobar resection versus lobectomy or more extensive resection for lung cancer patients with idiopathic pulmonary fibrosis. J Thorac Dis. 2016;8(5):977-84.

2. Tomassetti $\mathrm{S}$, et al. The impact of lung cancer on survival of idiopathic pulmonary fibrosis. Chest. 2015;147(1):157-64.

3. Ghatol A, Ruhl AP, Danoff SK. Exacerbations in idiopathic pulmonary fibrosis triggered by pulmonary and nonpulmonary surgery: a case series and comprehensive review of the literature. Lung. 2012;190(4):373-80.

4. King TE Jr, Pardo A, Selman M. Idiopathic pulmonary fibrosis. Lancet. 2011; 378(9807):1949-61.

5. Chuchalin AG. Idiopathic pulmonary fibrosis. Ter Arkh. 2000;72(3):5-12

6. Khali W, et al. Pathologic regulation of collagen I by an aberrant protein Phosphatase 2A/Histone Deacetylase C4/MicroRNA-29 signal Axis in idiopathic pulmonary fibrosis fibroblasts. Am J Respir Cell Mol Biol. 2015; 53(3):391-9

7. Romero $\mathrm{Y}$, et al. mTORC1 activation decreases autophagy in aging and idiopathic pulmonary fibrosis and contributes to apoptosis resistance in IPF fibroblasts. Aging Cell. 2016;15(6):1103-12.

8. Guiot J, Moermans C, Henket M, Corhay JL, Louis R. Blood biomarkers in idiopathic pulmonary fibrosis. Lung. 2017;195(3):273-80.

9. Zhao YY, Cheng XL, Lin RC. Lipidomics applications for discovering biomarkers of diseases in clinical chemistry. Int Rev Cell Mol Biol. 2014; 313:1-26.

10. Zhou X, et al. Identification of plasma lipid biomarkers for prostate cancer by lipidomics and bioinformatics. PLoS One. 2012;7(11):e48889.

11. Zehethofer $\mathrm{N}$, et al. Lipid analysis of airway epithelial cells for studying respiratory diseases. Chromatographia. 2015;78(5-6):403-13.

12. Simons K, Toomre D. Lipid rafts and signal transduction. Nat Rev Mol Cell Biol. 2000;1 (1):31-9.

13. Farooqui AA. Lipid mediators in the neural cell nucleus: their metabolism, signaling, and association with neurological disorders. Neuroscientist. 2009; 15(4):392-407.

14. Spiegel $\mathrm{S}$, Milstien $\mathrm{S}$. The outs and the ins of sphingosine-1-phosphate in immunity. Nat Rev Immunol. 2011;11(6):403-15.
15. Wood PL. Mass spectrometry strategies for clinical metabolomics and lipidomics in psychiatry, neurology, and neuro-oncology. Neuropsychopharmacology. 2014;39(1):24-33.

16. Min HK, Lim S, Chung BC, Moon MH. Shotgun lipidomics for candidate biomarkers of urinary phospholipids in prostate cancer. Anal Bioanal Chem. 2011;399(2):823-30.

17. Kim IC, et al. Lipid profiles for HER2-positive breast cancer. Anticancer Res. 2013;33(6):2467-72.

18. Graessler J, et al. Top-down lipidomics reveals ether lipid deficiency in blood plasma of hypertensive patients. PLoS One. 2009;4(7):e6261.

19. de Gomez Dumm NT, Giammona AM, Touceda LA, Raimondi C. Lipid abnormalities in chronic renal failure patients undergoing hemodialysis. Medicina (B Aires). 2001;61(2):142-6.

20. Yang $Y$, Uhlig $\mathrm{S}$. The role of sphingolipids in respiratory disease. Ther Adv Respir Dis. 2011;5(5):325-44.

21. Nixon GF. Sphingolipids in inflammation: pathological implications and potential therapeutic targets. Br J Pharmacol. 2009;158(4):982-93.

22. Masini E, et al. Ceramide: a key signaling molecule in a Guinea pig model of allergic asthmatic response and airway inflammation. J Pharmacol Exp Ther. 2008:324(2):548-57.

23. Teichgraber $V$, et al. Ceramide accumulation mediates inflammation, cell death and infection susceptibility in cystic fibrosis. Nat Med. 2008;14(4): 382-91.

24. Ley B, Brown KK, Collard HR. Molecular biomarkers in idiopathic pulmonary fibrosis. American journal of physiology. Lung Cell Mol Physiol. 2014;307(9): L681-91.

25. Yang K, Han X. Lipidomics: techniques, applications, and outcomes related to biomedical sciences. Trends Biochem Sci. 2016;41(11):954-69.

26. Schwudke D, Schuhmann K, Herzog R, Bornstein SR, Shevchenko A. Shotgun lipidomics on high resolution mass spectrometers. Cold Spring Harb Perspect Biol. 2011;3(9):a004614.

27. Yang K, Cheng H, Gross RW, Han X. Automated lipid identification and quantification by multidimensional mass spectrometry-based shotgun lipidomics. Anal Chem. 2009;81(11):4356-68.

28. Southam AD, et al. Drug redeployment to kill leukemia and lymphoma cells by disrupting SCD1-mediated synthesis of monounsaturated fatty acids. Cancer Res. 2015;75(12):2530-40.

29. McLaren $\mathrm{DG}$, et al. An ultraperformance liquid chromatography method for the normal-phase separation of lipids. Anal Biochem. 2011;414(2):266-72.

30. Sampaio JL, et al. Membrane lipidome of an epithelial cell line. Proc Natl Acad Sci U S A. 2011;108(5):1903-7.

31. Kuhn T, et al. Higher plasma levels of lysophosphatidylcholine 18:0 are related to a lower risk of common cancers in a prospective metabolomics study. BMC Med. 2016;14:13.

32. Piszcz J, et al. To treat or not to treat: metabolomics reveals biomarkers for treatment indication in chronic lymphocytic leukaemia patients. Oncotarget. 2016;7(16):22324-38.

33. Zhao YY, Lin RC. UPLC-MS(E) application in disease biomarker discovery: the discoveries in proteomics to metabolomics. Chem Biol Interact. 2014;215:7-16.

34. Wilson ID, et al. High resolution "ultra performance" liquid chromatography coupled to oa-TOF mass spectrometry as a tool for differential metabolic pathway profiling in functional genomic studies. J Proteome Res. 2005;4(2): 591-8.

35. Wang $C Z$, et al. Ultra-performance liquid chromatography and time-of-flight mass spectrometry analysis of ginsenoside metabolites in human plasma. Am J Chin Med. 2011;39(6):1161-71.

36. Raghu G, et al. An official ATS/ERS/JRS/ALAT statement: idiopathic pulmonary fibrosis: evidence-based guidelines for diagnosis and management. Am J Respir Crit Care Med. 2011;183(6):788-824.

37. Pedersen HK, et al. Human gut microbes impact host serum metabolome and insulin sensitivity. Nature. 2016;535(7612):376-81.

38. Fahy E, et al. A comprehensive classification system for lipids. J Lipid Res. 2005:46(5):839-61.

39. Ohnishi H, et al. Comparative study of KL-6, surfactant protein-a, surfactant protein-D, and monocyte chemoattractant protein-1 as serum markers for interstitial lung diseases. Am J Respir Crit Care Med. 2002;165(3):378-81.

40. Oikonomidi S, et al. Matrix metalloproteinases in respiratory diseases: from pathogenesis to potential clinical implications. Curr Med Chem. 2009;16(10): 1214-28.

41. Ollero $\mathrm{M}$, et al. Plasma lipidomics reveals potential prognostic signatures within a cohort of cystic fibrosis patients. J Lipid Res. 2011;52(5):1011-22. 
42. Goss V, Hunt AN, Postle AD. Regulation of lung surfactant phospholipid synthesis and metabolism. Biochim Biophys Acta. 2013;1831(2):448-58.

43. Frisardi V, Panza F, Seripa D, Farooqui T, Farooqui AA. Glycerophospholipids and glycerophospholipid-derived lipid mediators: a complex meshwork in Alzheimer's disease pathology. Prog Lipid Res. 2011;50(4):313-30.

44. Guerrera IC, et al. A novel lipidomic strategy reveals plasma phospholipid signatures associated with respiratory disease severity in cystic fibrosis patients. PLoS One. 2009;4(11):e7735.

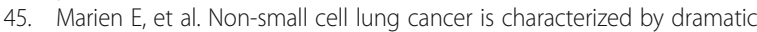
changes in phospholipid profiles. Int J Cancer. 2015;137(7):1539-48.

46. Wanders RJ, et al. Peroxisomal fatty acid alpha- and beta-oxidation in health and disease: new insights. Adv Exp Med Biol. 2003;544:293-302.

47. Lee ST, et al. Global changes in lipid profiles of mouse cortex, hippocampus, and hypothalamus upon p53 knockout. Sci Rep. 2016;6:36510.

48. Harvald EB, Olsen AS, Faergeman NJ. Autophagy in the light of sphingolipid metabolism. Apoptosis. 2015;20(5):658-70.

49. Gault CR, Obeid LM, Hannun YA. An overview of sphingolipid metabolism: from synthesis to breakdown. Adv Exp Med Biol. 2010;688:1-23.

50. Scarpa MC, et al. Ceramide expression and cell homeostasis in chronic obstructive pulmonary disease. Respiration. 2013;85(4):342-9.

51. Miao $\mathrm{H}$, et al. Plasma lipidomics reveal profound perturbation of glycerophospholipids, fatty acids, and sphingolipids in diet-induced hyperlipidemia. Chem Biol Interact. 2015;228:79-87.

52. Ishikawa $\mathrm{M}$, et al. Plasma and serum lipidomics of healthy white adults shows characteristic profiles by subjects' gender and age. PLoS One. 2014; 9(3):e91806.

53. Cai X, et al. Untargeted Lipidomic Profiling of Human Plasma Reveals Differences due to Race, Gender and Smoking Status. Metabolomics. 2014;4: 131). doi:10.4172/2153-0769.1000131.

\section{Submit your next manuscript to BioMed Central and we will help you at every step:}

- We accept pre-submission inquiries

- Our selector tool helps you to find the most relevant journal

- We provide round the clock customer support

- Convenient online submission

- Thorough peer review

- Inclusion in PubMed and all major indexing services

- Maximum visibility for your research

Submit your manuscript at www.biomedcentral.com/submit

C) Biomed Central 